\title{
Mucinous carcinoma is a predictive factor for the risk of open conversion from laparoscopic colectomy in colorectal cancer
}

\author{
Ah Jung Seo, Jung Kyoung Shin, Yoon Ah Park, Jung Wook Huh, Yong Beom Cho, Hee Cheol Kim, Seong Hyeon Yun, Woo Yong Lee \\ Department of Surgery, Samsung Medical Center, Sungkyunkwan University School of Medicine, Seoul, Korea
}

Purpose: Although laparoscopic surgery is widely accepted in the treatment of colorectal cancer, conversion to open surgery is associated with the rate of unfavorable outcomes. The aim of this study was to determine the factors associated with open conversion from laparoscopic surgery for colorectal cancer.

Methods: A total of 3,002 patients who underwent laparoscopic colectomy as an initial plan for the treatment of colorectal cancer located from the sigmoid colon to the rectum were retrospectively evaluated between January 2009 and December 2018 at Samsung Medical Center in Korea. Risk factors significantly associated with open conversion were determined using univariate and multivariate regression models.

Results: Among the 3,002 patients, open conversion was performed in 120 patients (4\%). Age $>60$ years (adjusted odds ratio [AOR], 2.370), preoperative bowel obstruction (AOR, 2.348), clinical T4 stage (AOR, 2.201), and serum carcinoembryonic antigen level $>5 \mathrm{ng} / \mathrm{mL}(A O R$, 2.289) were significantly associated with open conversion. Moreover, mucinous carcinoma was a significantly more frequent histopathologic type than adenocarcinoma (10.0\% vs. 3.2\%, $\mathrm{P}<0.001)$ in the open conversion group with an AOR of 2.549 (confidence interval, 1.259-5.159; $\mathrm{P}=0.009$ ).

Conclusion: The present study presented a novel finding, i.e. mucinous carcinoma as the histopathologic type could be an independent predictive factor for conversion from laparoscopic colectomy to open surgery. Identifying patients with mucinous carcinoma will help stratify the risk of open conversion preoperatively.

Keywords: Laparoscopic surgery, Colorectal neoplasms, Open conversion, Mucinous carcinoma

\section{INTRODUCTION}

Laparoscopic colorectal surgery is widely performed in the resec-

Received: Jun 27, 2019 Revised: Sep 7, 2019 Accepted: Sep 17, 2019

Correspondence to: Woo Young Lee

Department of Surgery, Samsung Medical Center, Sungkyunkwan University

School of Medicine, 81 Irwon-ro, Gangnam-gu, Seoul 06351, Korea

Tel: +82-2-3410-0261, Fax: +82-2-3410-6980

E-mail: Iwy555@skku.edu

ORCID: Ah Jung Seo (https://orcid.org/0000-0001-5830-9893), Jung Kyoung Shin (https://orcid.org/0000-0002-2298-8799), Yoon Ah Park (https://orcid.org/00000002-2328-1613), Jung Wook Huh (https://orcid.org/0000-0002-4485-0184), Yong Beom Cho (https://orcid.org/0000-0002-9944-4706), Hee Cheol Kim (https:// orcid.org/0000-0002-1610-5363), Seong Hyeon Yun (https://orcid.org/0000-00029591-4088), Woo Yong Lee (https://orcid.org/0000-0002-9558-9019)

Copyright @ 2019 Korean Society of Surgical Oncology

This is an Open Access article distributed under the terms of the Creative Commons Attribution Non-Commercial License (http://creativecommons.org/licenses/by-nc/4.0) which permits unrestricted non-commercial use, distribution, and reproduction in any medium, provided the original work is properly cited. tion of colorectal cancer owing to its lower complication rate, lower rate of perioperative morbidity, and shorter hospital stay compared with open surgery $[1,2]$. However, there have been concerns about the risk of conversion to open surgery during laparoscopic colorectal surgery [3].

Several studies have reported higher rates of postoperative morbidity from open conversion $[4,5]$. The risk factors associated with open conversion have been identified, including body mass index (BMI), American Society of Anesthesiologists (ASA) physical status classification, left-sided and low anterior resection, clinical T4 stage, acute surgery, metastatic setting, sex, age, and hospital volume $[3,6,7]$. However, whether the histologic findings of colorectal cancer affect the conversion of laparoscopic colorectal surgery to open surgery is not well established.

In colorectal cancer, almost $90 \%$ of cases are adenocarcinoma in histologic tumor subtypes. Among the other subtypes, mucinous carcinoma is observed in approximately $4 \%-19 \%$ of cases and more commonly found in the proximal colon [8]. Nevertheless, 
when mucinous carcinoma presents in the rectum, it behaves more aggressively [9]. A study suggests that the reason for aggressiveness when located in the rectum may be due to the anatomical effect of the pelvic cavity [10].

The purpose of the present study was to evaluate the factors associated with the risk of open conversion with respect to the pathologic types of colorectal cancers, especially those located in the sigmoid colon and rectum.

\section{METHODS}

\section{Patient inclusion criteria and data collection}

Patients who were initially planned to undergo laparoscopic surgery between January 2009 and December 2018 at Samsung Medical Center for colorectal cancer below the descending colon, including the sigmoid colon, rectosigmoid junction, and rectum, were retrospectively evaluated. This study was approved by the Institutional Review Board of Samsung Medical Center (IRB No. 2019-10-156) before data collection began. Data on patients' demographics, underlying diseases, prior abdominal surgery (PAS) history, preoperative bowel obstruction, carcinoembryonic antigen (CEA) serum levels measured before and after surgery, neoadjuvant concurrent chemoradiotherapy (CCRT) for rectal cancer, pathologic diagnosis of surgical specimens, pathologic stage of cancer, and cases of open conversion were collected from medical records. PAS was classified as major and minor PAS. Major PAS includes cases of abdominal surgery involving more than one abdominal quadrant. Minor PAS was defined as abdominal surgery involving one abdominal quadrant such as laparoscopic surgery with cholecystectomy and appendectomy, and benign gynecologic surgery [11]. Mucinous carcinoma is defined as a tumor with $>50 \%$ of extracellular mucin component confirmed through microscopic inspection $[12,13]$. Patients with lymphoma, familial adenomatous polyposis or hereditary nonpolyposis colorectal cancer, and rectal neuroendocrine tumor, as well as those who had surgical resection for recurrent colorectal cancer were excluded. The patients were categorized into two groups: a group in which conversion of laparoscopic surgery to open surgery was needed, and another group in which laparoscopic surgery was completed without conversion to open surgery.

\section{Determination of the need for conversion of laparoscopic surgery to open surgery}

Conversion is an intraoperative switch from a laparoscopic approach to an open abdominal approach. There are two subtypes of conversion: strategic conversion and reactive conversion. Strategic conversion means a standard laparotomy which is decided directly after the assessment of the feasibility of completing the procedure laparoscopically, the operative difficulty, or logistic consideration. It was not considered as a conversion when laparotomy chosen after a diagnostic laparoscopy (i.e., to assess the curability of the disease). Reactive conversion is the need for a laparotomy because of complications or operative difficulties after a considerable amount of dissection [14]. In this study, patients who met the criteria of one of the two subtypes were included in the conversion group. The reasons for conversion were categorized as follows: patient's physical environment, intraoperative bleeding, severe adhesion caused by a previous abdominal operation or with unknown cause, cancer invasion to adjacent organs, perforation, obstruction, advanced $\mathrm{N}$ stage, positive distal resection margin, problems related to the anastomosis site, peritoneal seeding, and desaturation after pneumoperitoneum. Conversion owing to the patient's physical environment was defined as when conversion was needed because of the patient's narrow pelvic cavity, anatomical variations, or underlying diseases such as liver cirrhosis or a huge benign ovarian mass. Open conversion owing to advanced $\mathrm{N}$ stage was based on the presence of suspicious metastatic lymph nodes beyond the surgical plane. Anastomosis-related problems that caused conversion from laparoscopy to open surgery included bowel color change or devascularization of the anastomosis site after the completion of the anastomosis procedure.

\section{Statistical analysis}

SPSS version 22.0 (IBM Corp., Armonk, NY, USA) was used for statistical analysis. The normality of data was tested using the Kolmogorov-Smirnov method. The independent $\mathrm{t}$-test was used to evaluate statistically significant differences between mean values of continuous variables. The chi-square test was performed to compare categorical variables. Values of $\mathrm{P}<0.05$ were considered statistically significant. Variables potentially related to the risk of open conversion with $\mathrm{P}<0.05$ on univariable analysis were included in multivariate analysis.

\section{RESULTS}

A total of 3,002 patients diagnosed with sigmoid or rectosigmoid junction or rectal cancer were initially planned to undergo laparoscopic colorectal cancer surgery. Of these patients, 2,882 (96\%) underwent laparoscopic completion colectomy for colorectal cancer and 120 underwent conversion to open colorectal surgery (conversion rate, 4\%). Among the 120 patients, 65 (54.1\%) were strategic conversion and 55 (45.9\%) were reactive conversion.

The demographic features of patients and preoperative clinical data including the location of the cancer, major or minor PAS, 
BMI, CCRT, and preoperative serum levels of CEA and carbohydrate antigen 19-9 (CA19-9) are summarized in Table 1. The median age was 66 and 60 years in the conversion group and non-conversion group, respectively, and was significantly higher in the conversion group (mean \pm standard deviation: $66.68 \pm 13.4$ years vs. $60.28 \pm 11.66$ years, $\mathrm{P}<0.001)$. There were no differences in sex $(\mathrm{P}=0.771)$ or cancer location between the two groups (Table 1$)$.

PAS ( $\mathrm{P}=0.617)$ showed no difference in the conversion group and the non-conversion group. In addition, major PAS and minor PAS had no significant differences $(\mathrm{P}=0.138$ and $\mathrm{P}=0.824$, respectively). Preoperative serum level of CA19-9 $(\mathrm{P}=0.870)$, preoperative CCRT $(\mathrm{P}=0.87)$, and underlying diseases including hyper- tension $(\mathrm{P}=0.366)$ and diabetes mellitus $(\mathrm{P}=0.209)$ appeared to have no significant differences between the two groups.

On the other hand, the presence of preoperative obstruction (37.5\% vs. $13.1 \%, \mathrm{P}<0.001)$ and clinical T4 stage $(29.2 \%$ vs. $10.2 \%$, $\mathrm{P}<0.001$ ) were more frequently observed in the open conversion group than in the laparoscopic completion group. Additionally, 27 (22.5\%) patients in the open conversion group and 421 (14.6\%) patients in the laparoscopic completion group had N2 stage, with a statistically significant difference $(\mathrm{P}=0.019)$. The preoperative serum level of CEA tended to be more elevated in patients who underwent conversion from laparoscopic to open surgery than in those who underwent laparoscopic completion $(\mathrm{P}=0.005)$. With

Table 1. Patients' demographics and characteristics

\begin{tabular}{|c|c|c|c|}
\hline Patients characteristics & $\begin{array}{l}\text { Open conversion } \\
\quad(n=120)\end{array}$ & $\begin{array}{l}\text { Laparoscopic completion } \\
\qquad(n=2,882)\end{array}$ & P-value \\
\hline Sex & & & 0.771 \\
\hline Male & $74(61.7)$ & $1739(60.3)$ & \\
\hline Female & 46 (38.3) & $1143(39.7)$ & \\
\hline Age (yr) & $66.7 \pm 13.4$ & $60.3 \pm 11.7$ & $<0.001$ \\
\hline Body mass index $\left(\mathrm{kg} / \mathrm{m}^{2}\right)$ & $24.2 \pm 4.3$ & $24.1 \pm 13.8$ & 0.959 \\
\hline \multicolumn{4}{|l|}{ Location of cancer } \\
\hline Sigmoid colon & $42(35.0)$ & $1,110(38.5)$ & 0.337 \\
\hline Rectosigmoid junction & $20(16.7)$ & 525 (18.2) & 0.666 \\
\hline Rectum & $58(48.3)$ & $1,247(43.3)$ & 0.202 \\
\hline Prior abdominal surgery & $33(27.5)$ & $734(25.5)$ & 0.617 \\
\hline Major PAS & 8 (6.7) & $108(3.7)$ & 0.138 \\
\hline Minor PAS & $25(20.8)$ & $625(25.7)$ & 0.824 \\
\hline Preoperative CEA (ng/mL) & $47.2 \pm 149.4$ & $7.7 \pm 49.5$ & 0.005 \\
\hline Preoperative CA19-9 (U/mL) & $86.8 \pm 308.1$ & $151.5 \pm 3,661.8$ & 0.870 \\
\hline Preoperative CCRT ${ }^{\mathrm{a})}$ & $24(41.3)$ & 441 (35.3) & 0.870 \\
\hline HTN & $44(36.7)$ & $1,013(40.8)$ & 0.366 \\
\hline DM & $28(23.3)$ & 465 (18.7) & 0.209 \\
\hline \multicolumn{4}{|l|}{ Pathology } \\
\hline Adenocarcinoma & 104 (86.7) & 2,678 (92.9) & 0.004 \\
\hline Mucinous carcinoma & $12(10.0)$ & $92(3.2)$ & $<0.001$ \\
\hline Squamous cell carcinoma & $3(2.5)$ & 0 & - \\
\hline Signet ring cell carcinoma & $1(0.8)$ & $30(1.04)$ & $>0.990$ \\
\hline \multicolumn{4}{|l|}{ Clinical T category } \\
\hline $\mathrm{T} 1$ & $10(8.3)$ & 555 (19.3) & 0.003 \\
\hline $\mathrm{T} 2$ & $5(4.2)$ & $479(16.6)$ & $<0.001$ \\
\hline T3 & 70 (58.3) & $1.554(53.9)$ & 0.344 \\
\hline $\mathrm{T} 4$ & $35(29.2)$ & 293 (10.2) & $<0.001$ \\
\hline \multicolumn{4}{|l|}{ Clinical N category } \\
\hline No & 59 (49.2) & $1,624(56.4)$ & 0.117 \\
\hline N1 & $34(28.3)$ & $834(29)$ & 0.881 \\
\hline N2 & 27 (22.5) & $421(14.6)$ & 0.019 \\
\hline
\end{tabular}

Values are presented as number (\%) or mean \pm standard deviation.

PAS, prior abdominal surgery; CEA, carcinoembryonic antigen; CA19-9, carbohydrate antigen 19-9; CCRT, concurrent chemoradiotherapy; HTN, hypertension; DM, diabetes mellitus.

a) Preoperative CCRT was evaluated only in the patients with rectal cancer. 
respect to the histologic cancer subtypes (adenocarcinoma or mucinous carcinoma), there were major differences between the two groups. Mucinous carcinoma was diagnosed in 10 patients (10\%) in the open conversion group compared with 92 patients (3.2\%) in the laparoscopic completion group $(\mathrm{P}<0.001)$, as shown in Table 1 .

The reasons for conversion are summarized in Table 2. Cancer invasion to adjacent organs was the most frequent reason for conversion (43.3\%), followed by adhesion (12.5\%), intraoperative bleeding (10.8\%), and obstruction (10\%). Further, there were other

Table 2. Reasons for conversion

\begin{tabular}{lc}
\hline Reason for conversion & No. of patients (\%) \\
\hline Invasion & $52(43.3)$ \\
Adhesions $^{\mathrm{a})}$ & $15(12.5)$ \\
Intraoperative bleeding & $13(10.8)$ \\
Obstruction & $12(10)$ \\
Peritoneal seeding & $7(5.8)$ \\
Patient's physical environment ${ }^{\mathrm{b})}$ & $6(5)$ \\
Obesity & $4(4.3)$ \\
Anastomosis site-related problems ${ }^{\mathrm{c}}$ & $4(4.3)$ \\
Metastatic lymph nodes & $3(2.5)$ \\
Perforation & $2(1.7)$ \\
Positive distal resection margin & $1(0.8)$ \\
Desaturation after pneumoperitoneum & $1(0.8)$ \\
Total & $120(100)$ \\
\hline
\end{tabular}

${ }^{a)}$ Adhesions due to previous abdominal operations or intra-abdominal adhesions with unknown reasons. ${ }^{b}$ The patient's physical environment includes a narrow pelvis, anatomical variations, and underlying diseases such as liver cirrhosis or a huge mass in the ovary. ${ }^{\mathrm{c}}$ Anastomosis site-related problems include bowel color change or devascularization of the anastomosis site after the completion of the anastomosis procedure. factors for open conversion during laparoscopic surgery, including peritoneal seeding during laparoscopic exploration in seven patients (5.8\%), a narrow pelvis or a friable and easy-to-bleed tissue due to underlying liver cirrhosis, or a fatty mesentery, or a huge ovarian mass that prevented the laparoscopic procedure in six patients (5\%), a bowel color change or a devascularization of the anastomosis site after anastomosis in four patients $(4.3 \%)$, and suspicious metastatic lymph nodes found beyond the surgical field (e.g., para-aortic or pelvic lesions) in three patients (2.5\%) (Table 2).

Univariate and multivariate analyses were performed to evaluate the risk factors for conversion from laparoscopic to open colectomy (Table 3). Univariate analysis showed that age $\geq 60$ years (odds ratio $[\mathrm{OR}], 2.407$; 95\% confidence interval [CI], 1.631-3.551; $\mathrm{P}<0.001$ ), preoperative CEA serum level $>5.0 \mathrm{ng} / \mathrm{mL}$ (OR, 3.423; 95\% CI, 2.332-5.025; $\mathrm{P}<0.001$ ), clinical T4 stage (OR, 3.637; 95\% CI, 2.409-5.490; $\mathrm{P}<0.001$ ), N2 stage (OR, 1.695; 95\% CI, 1.0912.634; $\mathrm{P}=0.019)$, bowel obstruction (OR, 3.987; 95\% CI, 2.7125.860; $\mathrm{P}<0.001)$, and mucinous carcinoma as the histologic type (OR, 3.370; 95\% CI, 1.792-6.336; $\mathrm{P}<0.001$ ) had higher odds ratios in the open conversion group than in the laparoscopic completion group. Preoperative CCRT did not seem to increase the risk for open conversion (OR, $0.775 ; 95 \% \mathrm{CI}, 0.454-1.324 ; \mathrm{P}=0.870$ ), as it showed no significant difference between the two groups (Table 3 ).

The risk of conversion was further analyzed with respect to the associations between variables (Table 3). Among them, the risk of conversion was the highest with the mucinous carcinoma histology, with an adjusted odds ratio (AOR) of 2.549 (95\% CI, 1.2595.159; $\mathrm{P}=0.009)$. Moreover, the odds of open conversion were higher with bowel obstruction (AOR, 2.348; 95\% CI, 1.517-3.634; $\mathrm{P}<0.001$ ), preoperative CEA serum level $>5.0 \mathrm{ng} / \mathrm{mL}$ (AOR,

Table 3. Risk factors of open conversion from laparoscopic surgery in univariable and multivariable analyses of the cohort ( $n=3,002)$

\begin{tabular}{|c|c|c|c|c|}
\hline & \multicolumn{2}{|c|}{ Univariable analysis } & \multicolumn{2}{|c|}{ Multivariable analysis } \\
\hline & OR $(95 \% \mathrm{Cl})$ & P-value & OR $(95 \% \mathrm{Cl})$ & P-value \\
\hline \multicolumn{5}{|l|}{ Age } \\
\hline$\geq 60 \mathrm{yr}$ & $2.407(1.631-3.551)$ & $<0.001$ & $2.370(1.584-3.545)$ & $<0.001$ \\
\hline \multicolumn{5}{|l|}{$<60 \mathrm{yr}$} \\
\hline Preoperative $\mathrm{CCRT}^{\text {a) }}$ & $0.775(0.454-1.324)$ & 0.870 & & \\
\hline \multicolumn{5}{|l|}{ Preoperative CEA level } \\
\hline$\geq 5.0 \mathrm{ng} / \mathrm{mL}$ & $3.423(2.332-5.025)$ & $<0.001$ & $2.289(1.510-3.470)$ & $<0.001$ \\
\hline \multicolumn{5}{|l|}{$<5.0$ ng/mL } \\
\hline Clinical T4 stage & $3.637(2.409-5.490)$ & $<0.001$ & $2.201(1.371-3.533)$ & 0.001 \\
\hline Clinical N2 stage & $1.695(1.091-2.634)$ & 0.019 & $1.002(0.615-1.634)$ & 0.993 \\
\hline Bowel obstruction & $3.987(2.712-5.860)$ & $<0.001$ & $2.348(1.517-3.634)$ & $<0.001$ \\
\hline Mucinous carcinoma histology & $3.370(1.792-6.336)$ & $<0.001$ & $2.549(1.259-5.159)$ & 0.009 \\
\hline Adenocarcinoma histology & $0.462(0.271-0.786)$ & 0.004 & & \\
\hline
\end{tabular}

$\mathrm{OR}$, odds ratio; $\mathrm{Cl}$, confidence interval; CCRT, concurrent chemoradiotherapy; $C E A$, carcinoembryonic antigen.

a) Preoperative CCRT was evaluated only in the patients with rectal cancer. 
2.289; 95\% CI, 1.510-3.470; $\mathrm{P}<0.001$ ), clinical T4 stage (AOR, 2.201; 95\% CI, 1.371-3.533; $\mathrm{P}<0.001$ ), and age (AOR, 2.370; 95\% $\mathrm{CI}, 1.584-3.545 ; \mathrm{P}<0.001)$. However, clinical N2 stage was not associated with the risk of conversion.

\section{DISCUSSION}

In 2016, Stormark et al. [7] studied a large national patient cohort and confirmed the safety of laparoscopic colectomy and its similar long-term survival to that of open surgery. A recent study showed that the use of laparoscopic colectomy has increased to $>80 \%$ and the conversion rate has decreased from $12 \%$ to $8 \%$ in colorectal cancer between 2011 and 2015. The decrease in conversion was significantly related to the increased laparoscopic volume in hospitals [15]. We observed that the conversion rate for sigmoid colon, rectosigmoid colon, and rectal cancer was 4.0\% between 2009 and 2018.

We found that the most frequent reason for conversion was invasion of cancer to adjacent tissues or organs, which accounts for nearly $50 \%$. Allaix et al. [16] reviewed all literature on cases of open conversion during laparoscopic resection for both colon cancer and rectal cancer for all years up to March 2016, and the most frequent reasons for conversion were also cancer-related factors. The other causes they reported were adhesion, obesity, or anatomic-related factors, which were similar to the results of our study.

Although several previous studies reported that male sex and obesity increased the risk of conversion [1,4], sex and BMI were not associated with conversion in this study. Some patients underwent conversion from laparoscopic to open surgery because of adhesions from previous abdominal operations. Kim et al. [11] found that major PAS and minor PAS presented different results. Patients with a history of major PAS showed a higher conversion rate than those with no PAS. In contrast, patients with a history of minor PAS and those with no PAS showed no difference in conversion rate. In our results, major PAS had no statistically significant difference between the conversion group and the laparoscopic completion group.

There have been some efforts to clarify the relationship between neoadjuvant chemoradiotherapy and technical difficulties in laparoscopic surgery for rectal cancer $[17,18]$. A study examined the effect of preoperative chemoradiotherapy in laparoscopic surgery for rectal cancer, and found no difference in conversion rate between the preoperative chemoradiotherapy group and the surgery only group ( $18.8 \%$ and $15.4 \%$, respectively; $\mathrm{P}=0.392)$ [19]. Correspondingly, we observed that preoperative chemoradiotherapy had no effect on the conversion from laparoscopic to open surgery. Because some studies found that high ASA scores increased the risk of conversion $[3,6]$, we also investigated patients with hypertension or diabetes mellitus. However, hypertension and diabetes mellitus had no individual effect on the conversion rate.

After multivariate analyses, we found five factors that could be independent predictors of conversion from laparoscopic to open surgery. Although higher age was not a risk factor for open conversion in previous studies [6,7], patients aged $>60$ years had an almost 2-fold higher conversion risk in this study. Stormark et al. [7] and Tekkis et al. [3] already confirmed that higher $\mathrm{T}$ and $\mathrm{N}$ stages were associated with conversion from laparoscopic to open surgery. We also identified that patients with clinical T4 stage had a higher risk of conversion (AOR, 2.2). However, clinical N2 stage was not an individual risk factor in our study. Preoperative obstruction was also a strong risk factor that significantly affected the conversion risk (AOR, 2.3) in our data. Several studies had reported that emergent colorectal resection had a higher conversion rate than elective colorectal resection $[7,20]$.

There have been no reports on the relationship between preoperative serum CEA and CA19-9 levels and the conversion risk in laparoscopic colorectal resection for colorectal cancer. Comparing the laparoscopic completion group and the open conversion group, we confirmed that preoperative serum CEA serum level tended to be much higher in the open conversion group. A $>5.0 \mathrm{ng} / \mathrm{mL}$ serum CEA level was an independent risk factor with an AOR of 2.3. Although higher serum levels of both CEA and CA19-9 are related to the severity of colorectal cancer [21], the level of serum CA19-9 was not a risk factor for conversion in our findings.

In this study, mucinous carcinoma was diagnosed in 12 patients $(10 \%)$ in the open conversion group and in 92 patients (3.2\%) in the laparoscopic completion group $(\mathrm{P}<0.001)$. In addition, we observed that mucinous carcinoma as the histopathologic type was an independent risk factor with an AOR of 2.5. There have been no studies on the association between the histologic type of cancer and the risk of conversion. Mucinous carcinoma is diagnosed in $4 \%$ to $15 \%$ of all patients with colorectal cancer [22]. This histologic type is much rarer in the rectum [23] and more frequently found in the proximal colon [24]. Mucinous carcinoma is diagnosed if the tumor cells produce mucin that aggregates into pools or lakes, occupying $>50 \%$ of the tumor [25]. As the definition of mucinous carcinoma has varied according to the amount of mucus produced by neoplastic cells within the rectum over the years, mucinous tumors of the rectum are less well understood than non-mucinous tumors [10]. Several studies have observed that mucin has a pressure effect that causes dissemination of cancer cells into the peritoneal cavity [26-28]. Moreover, compared with adenocarcinoma, mucinous carcinoma is more frequently found at a higher disease stage at presentation [9,29]. Hyngstrom et al. [9] found that muci- 
Table 4. The details for the patients with mucinous carcinoma who were converted open colectomy from laparoscopic colectomy

\begin{tabular}{cclll}
\hline Case & Sex/age $(\mathrm{yr})$ & \multicolumn{1}{c}{ Diagnosis } & \multicolumn{1}{c}{ Reason for conversion } & Pathology \\
\hline 1 & $\mathrm{~F} / 84$ & Rectosigmoid junction cancer & Adhesion with abdominal wall & Mucinous carcinoma \\
2 & $\mathrm{~F} / 79$ & Rectal cancer & Suspicious of uterus invasion & Mucinous carcinoma \\
3 & $\mathrm{M} / 83$ & Sigmoid colon cancer & Severe adhesion with bladder and ureter & Mucinous carcinoma \\
4 & $\mathrm{M} / 71$ & Rectosigmoid junction cancer & Invasion of prostate, seminal vesicle and lateral abdominal wall & Mucinous carcinoma \\
5 & $\mathrm{M} / 37$ & Rectal cancer & Adhesion or invasion to retroperitoneum & Mucinous carcinoma \\
6 & $\mathrm{M} / 52$ & Sigmoid colon cancer & Adhesion or invasion to retroperitoneum & Mucinous carcinoma \\
7 & $\mathrm{M} / 53$ & Rectosigmoid junction cancer & Invasion to bladder, prostate, seminal vesicle, small bowel & Mucinous carcinoma \\
& & & Infiltration and inflammatory change of pelvic organ and tissue & Mucinous carcinoma \\
8 & $\mathrm{M} / 62$ & Rectal cancer & Invasion to adjacent organ with adhesion and fibrotic change & Mucinous carcinoma \\
10 & $\mathrm{M} / 82$ & Sigmoid colon cancer & Adhesion or invasion of peritoneum & Mucinous carcinoma \\
11 & $\mathrm{~F} / 42$ & Rectal cancer & Adhesion with peritoneum & Mucinous carcinoma \\
12 & $\mathrm{M} / 64$ & Sigmoid colon cancer & Invasion to adjacent organ & Mucinous carcinoma \\
\hline
\end{tabular}

F, female; $M$, male.

nous cancer located in the rectum showed worse survival outcomes with a hazard ratio (HR) of death of 1.22, but not for those located in the colon (HR, 1.03). Chand et al. [10] explained that mucinous carcinoma in the rectum has a more aggressive behavior than mucinous carcinoma in the colon because of the anatomical location and the immediate effect of the narrow pelvic cavity. In the present study, the association between the mucinous histologic type and open conversion in low colorectal cancer can be explained by the unique clinicopathologic behavior of mucin. Table 4 shows the clinical reason for open conversion in 12 patients in whom the histologic type of cancer was mucinous carcinoma. All 12 cases were converted to open laparotomy from laparoscopic colectomy due to invasion or adhesion. Identifying patients with mucinous carcinoma in preoperative colonoscopy biopsy or those suspicious of having mucinous carcinoma in preoperative magnetic resonance imaging will aid the prediction of the possibility of open conversion by understanding the unique features of mucinous carcinoma.

The predictive factors we investigated would allow surgeons to prepare for cases of open conversion by stratifying the conversion risk. However, it remains difficult to draw a proper conclusion owing to the inherent limitations of a retrospective study and the small sample size in subgroup analysis. To strengthen our results, further evaluations of mucinous carcinoma located in proximal colon and multicenter studies will be required. In addition, further studies on short- and long-term outcomes comparing the laparoscopic completion group and the open conversion group of patients with mucinous carcinoma would provide more evidence for determining the treatment plan.

In conclusion, this study demonstrated that age $>60$ years, clini- cal T4 stage, bowel obstruction by the cancer, CEA serum level $>5.0 \mathrm{ng} / \mathrm{mL}$, and mucinous carcinoma as the histologic type could be independent predictors of conversion from laparoscopic to open colectomy in colorectal cancer.

\section{CONFLICT OF INTEREST}

No potential conflict of interest relevant to this article was reported.

\section{REFERENCES}

1. 2017 and 2015 European Society of Coloproctology (ESCP) collaborating groups. The impact of conversion on the risk of major complication following laparoscopic colonic surgery: an international, multicentre prospective audit. Colorectal Dis 2018;20 Suppl 6:69-89.

2. Rabasova M, Martinek L. Conversion risk factors in laparoscopic colorectal surgery. Wideochir Inne Tech Maloinwazyjne 2012;7: 240-5.

3. Tekkis PP, Senagore AJ, Delaney CP. Conversion rates in laparoscopic colorectal surgery: a predictive model with, 1253 patients. Surg Endosc 2005;19:47-54.

4. Belizon A, Sardinha CT, Sher ME. Converted laparoscopic colectomy: what are the consequences? Surg Endosc 2006;20:947-51.

5. Kang CY, Chaudhry OO, Halabi WJ, Nguyen V, Carmichael JC, Stamos MJ, et al. Outcomes of laparoscopic colorectal surgery: data from the Nationwide Inpatient Sample 2009. Am J Surg 2012;204: 952-7.

6. Kuhry E, Bonjer HJ, Haglind E, Hop WC, Veldkamp R, Cuesta MA, et al. Impact of hospital case volume on short-term outcome 
after laparoscopic operation for colonic cancer. Surg Endosc 2005; 19:687-92.

7. Stormark K, Soreide K, Soreide JA, Kvaloy JT, Pfeffer F, Eriksen MT, et al. Nationwide implementation of laparoscopic surgery for colon cancer: short-term outcomes and long-term survival in a population-based cohort. Surg Endosc 2016;30:4853-64.

8. Hamilton CH, Hamilton WW, Hamilton WH. Polypoid lesions of the colon and rectum: a discussion of recognition and management. Ohio State Med J 1965;61:129-33.

9. Hyngstrom JR, Hu CY, Xing Y, You YN, Feig BW, Skibber JM, et al. Clinicopathology and outcomes for mucinous and signet ring colorectal adenocarcinoma: analysis from the National Cancer Data Base. Ann Surg Oncol 2012;19:2814-21.

10. Chand M, Yu S, Swift RI, Brown G. Mucinous carcinoma of the rectum: a distinct clinicopathological entity. Tech Coloproctol 2014;18:335-44.

11. Kim IY, Kim BR, Kim YW. Impact of prior abdominal surgery on rates of conversion to open surgery and short-term outcomes after laparoscopic surgery for colorectal cancer. PLoS One 2015;10: e0134058.

12. Langner C, Harbaum L, Pollheimer MJ, Kornprat P, Lindtner RA, Schlemmer A, et al. Mucinous differentiation in colorectal cancer: indicator of poor prognosis? Histopathology 2012;60:1060-72.

13. Zhao J, Xu J, Zhang R. Clinical and prognostic significance of pathological and inflammatory markers in mucinous rectal cancer patients receiving neoadjuvant chemoradiotherapy and curative surgery. Med Sci Monit 2017;23:4826-33.

14. Blikkendaal MD, Twijnstra AR, Stiggelbout AM, Beerlage HP, Bemelman WA, Jansen FW. Achieving consensus on the definition of conversion to laparotomy: a Delphi study among general surgeons, gynecologists, and urologists. Surg Endosc 2013;27:4631-9.

15. de Neree Tot Babberich MPM, van Groningen JT, Dekker E, Wiggers T, Wouters MWJM, Bemelman WA, et al. Laparoscopic conversion in colorectal cancer surgery; is there any improvement over time at a population level? Surg Endosc 2018;32:3234-46.

16. Allaix ME, Furnee EJ, Mistrangelo M, Arezzo A, Morino M. Conversion of laparoscopic colorectal resection for cancer: what is the impact on short-term outcomes and survival? World J Gastroenterol 2016;22:8304-13.

17. Denoya P, Wang H, Sands D, Nogueras J, Weiss E, Wexner SD. Short-term outcomes of laparoscopic total mesorectal excision fol- lowing neoadjuvant chemoradiotherapy. Surg Endosc 2010;24: 933-8.

18. Kang SB, Park JW, Jeong SY, Nam BH, Choi HS, Kim DW, et al. Open versus laparoscopic surgery for mid or low rectal cancer after neoadjuvant chemoradiotherapy (COREAN trial): short-term outcomes of an open-label randomised controlled trial. Lancet Oncol 2010;11:637-45.

19. Denost Q, Laurent C, Paumet T, Quintane L, Martenot M, Rullier E. Laparoscopic surgery for rectal cancer: preoperative radiochemotherapy versus surgery alone. Surg Endosc 2012;26:1878-83.

20. Masoomi H, Moghadamyeghaneh Z, Mills S, Carmichael JC, Pigazzi A, Stamos MJ. Risk factors for conversion of laparoscopic colorectal surgery to open surgery: does conversion worsen outcome? World J Surg 2015;39:1240-7.

21. Stojkovic Lalosevic M, Stankovic S, Stojkovic M, Markovic V, Dimitrijevic I, Lalosevic J, et al. Can preoperative CEA and CA19-9 serum concentrations suggest metastatic disease in colorectal cancer patients? Hell J Nucl Med 2017;20:41-5.

22. Du W, Mah JT, Lee J, Sankila R, Sankaranarayanan R, Chia KS. Incidence and survival of mucinous adenocarcinoma of the colorectum: a population-based study from an Asian country. Dis Colon Rectum 2004;47:78-85.

23. Kang H, O'Connell JB, Maggard MA, Sack J, Ko CY. A 10-year outcomes evaluation of mucinous and signet-ring cell carcinoma of the colon and rectum. Dis Colon Rectum 2005;48:1161-8.

24. Verhulst J, Ferdinande L, Demetter P, Ceelen W. Mucinous subtype as prognostic factor in colorectal cancer: a systematic review and meta-analysis. J Clin Pathol 2012;65:381-8.

25. Jass JR, Sobin LH, Watanabe H. The World Health Organization's histologic classification of gastrointestinal tumors: a commentary on the second edition. Cancer 1990;66:2162-7.

26. Symonds DA, Vickery AL. Mucinous carcinoma of the colon and rectum. Cancer 1976;37:1891-900.

27. Parham D. Colloid carcinoma. Ann Surg 1923;77:90-105.

28. Sugarbaker PH. Mucinous colorectal carcinoma. J Surg Oncol 2001;77:282-3.

29. Hugen N, Verhoeven RH, Radema SA, de Hingh IH, Pruijt JF, Nagtegaal ID, et al. Prognosis and value of adjuvant chemotherapy in stage III mucinous colorectal carcinoma. Ann Oncol 2013;24: 2819-24. 